Classification

Physics Abstracts

24.75

\title{
High resolution measurement of fragment mass-charge-kinetic energy correlations in the cold fragmentation region of thermal fission of ${ }^{235} \mathbf{U}$
}

\author{
C. Signarbieux \\ Département de Physique Nucléaire, C.E.N. Saclay, 91191 Gif-sur-Yvette Cedex, France
}

G. Simon, J. Trochon and F. Brisard

Service de Physique et Techniques Nucléaires, C.E.N. de Bruyères-le-Châtel, B.P. no 12 , 91680 Bruyères-le-Châtel, France

(Reçu le 23 août 1985, accepté le 8 octobre 1985)

\begin{abstract}
Résumé. - Les performances d'une double chambre d'ionisation à grilles contenant une cible fissile extrêmement mince ont été explorées dans le but d'étudier le phénomène de fragmentation froide; l'énergie cinétique primaire des fragments est mesurée avec une résolution bien meilleure que $300 \mathrm{keV}$, la séparation des masses est totale et, pour chacune d'elles, une bonne séparation des charges est obtenue. Une discussion de l'évolution de la distribution des charges en fonction de l'énergie cinétique totale des fragments est faite pour le rapport de masses 132/104.
\end{abstract}

\begin{abstract}
The performance of a back-to-back gridded ionization chamber containing an extremely thin fissile target has been investigated for studying the cold fragmentation phenomenon : primary fragment kinetic energy is measured with a resolution much better than $300 \mathrm{keV}$; mass separation is total and for each mass, a good charge separation is obtained. The evolution of the charge distribution as a function of the fragments' total kinetic energy is discussed for the mass-ratio 132/104.
\end{abstract}

It has been known for quite a while now that in thermal neutron induced fission of ${ }^{235} \mathrm{U},{ }^{233} \mathrm{U}$ and ${ }^{239} \mathrm{Pu}$, a small fraction of events (about $3 \%$ ) is produced with no prompt neutron emission [1]. But it was recently demonstrated experimentally $[2,3]$ that such events do not only result from gamma-neutron competition but also correspond to fragmentations into two fragments, each of them having an excitation energy less than the neutron binding energy. By studying the massenergy correlations of the latter category of events, the authors found three quite unexpected properties :

- The phenomenon is not at all restricted to specific couples of fragments but is rather general (only symmetric and far-asymmetric mass regions being excluded).

- For many mass-ratios, the total kinetic energy of the fragments can reach values close to (if not equal to) the highest allowed $Q$-value, thus providing evidence for extremely compact scission configurations necessarily formed by two juxtaposed fragments, each being in (or near) 
its respective ground state. This particular fragmentation was subsequently referred to as « cold fragmentation $"$.

- The mass distributions do not show any systematic even-odd effect up to the extinction of the yield. This last property, however, conflicts with the strong even-odd effect observed in the charge distribution [4], and hence suggests a different behaviour for the neutron and proton systems [5].

The purpose of the experiment reported here is to investigate thoroughly this latter question by measuring the fragment mass-charge-energy correlations in the cold fragmentation region.

The back-to-back Frisch gridded ionization chamber was chosen as detection system for the following reasons :

1) Correlated measurements on both complementary fragments present the important advantage of determining the mass- and charge-ratios rather than individual masses and charges : the spacing between two adjacent ratios being twice that observed for individual masses and charges, because $\left(A_{1}+1\right)$ and $\left(Z_{1}+1\right)$ are necessarily correlated to $\left(A_{2}-1\right)$ and $\left(Z_{2}-1\right)$ in the cold fragmentation region.

2) Recently, Oed et al. [6] have shown that ionization chambers, when operated with light gases, exhibit a surprisingly good energy resolution for fission fragments. Moreover the same authors have also proposed a very simple method to analyse in an axial ionization chamber the charge distribution of fission fragments, provided they are properly selected in mass and kinetic energy [7].

3) In a back-to-back ionization chamber, the fissile target is located inside the chamber, thus avoiding the straggling effects in entrance windows of any time-of-flight system.

The gridded ionization chamber used and its associated electronics were quite conventional and we mention only the relevant features here : the detector was run with pure $\mathrm{CH}_{4}$ gas and an exceedingly thin target of $3 \mu \mathrm{g} / \mathrm{cm}^{2}$ of ${ }^{235} \mathrm{UF}_{4}$ evaporated on a $5 \mu \mathrm{g} / \mathrm{cm}^{2}$ carbon backing. For each fission event detected in coincidence in the two halves of the chamber, the following parameters were measured :

$-X_{1}$ and $X_{2}$, the anode pulse heights which are, as a first approximation, proportional to the energies deposited in the gas by either of the fragments.

$-T_{1}$ and $T_{2}$, the time differences between the beginning of the cathode pulse and the beginning of the cathode pulse and the beginning of the anode pulses (the actual three trigger thresholds have been set at about $6 \mathrm{MeV}$ ). It follows that $T_{1}$ and $T_{2}$ are a measurement of the projections of the fragment track lengths $R_{1}$ and $R_{2}$ along the electric field direction (see Fig. 1). If $V$ is the electron drift velocity, $d$ the cathode-grid distance, $\theta$ and $R$ the emission angle and the range of a fragment, then :

$$
T=\frac{d-R \cos \theta}{V}
$$

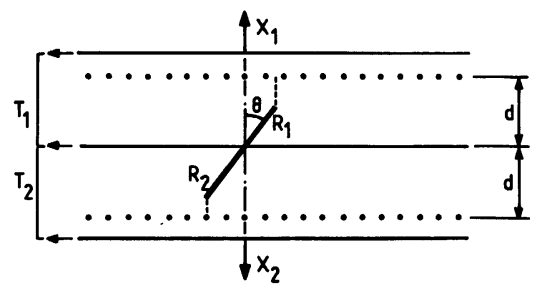

Fig. 1. - Diagram of the detection system. 
It is known that for ions with fixed mass and kinetic energy (velocity), the mean range $R$ then only depends on the nuclear charge $Z$.

The data analysis is rather straighforward :

1) The mass ratio $A_{1} / A_{2}$ is simply given by the $X_{1}-X_{2}$ correlation which reflects the momentum conservation law, which is strictly valid for events with no neutron emission.

2) When the mass-ratio is determined, the total kinetic energy of both fragments TKE is deduced from the kinetic energy of the fragment emerging from the deposit side (the fragment least affected by the energy straggling inside the target). It should be noted here that the absolute energy calibration is a problem not yet resolved and the values of the kinetic energy indicated in this paper have been deduced from data given in reference [8], as explained in reference [9].

3) For events with fixed $A_{1} / A_{2}$ and TKE, the sum of the two ranges $\left(R_{1}+R_{2}\right)$ can be considered, as a first approximation, constant. According to the evident relationship :

$$
\left(R_{1}+R_{2}\right) \cos \theta=V\left[2 d / V-\left(T_{1}+T_{2}\right)\right]
$$

the angular dependence is then simply given by the rectangular distribution of $\left(T_{1}+T_{2}\right)$ which reflects the isotropic distribution of the fragments in the laboratory system.

4) Finally, for fixed $A_{1} / A_{2}$ and TKE events selected to fall inside a cone centred at $\theta=0^{\circ}$, the charge-ratio distribution corresponds to the $R_{1} / R_{2}$ distribution defined by the second evident relationship :

$$
\frac{R_{2}}{R_{1}}=\frac{d / V-T_{2}}{d / V-T_{1}}
$$

where $d / V$ represents the electron drift time between the cathode and the grids.

The experiment, performed at the Saclay ORPHEE reactor, used a rather modest thermal neutron beam intensity of about $8 \times 10^{7} \mathrm{n} / \mathrm{cm}^{2} / \mathrm{s}$, giving a fission rate of roughly 100 counts/s. During a 4 day run, approximately $1.1 \times 10^{6}$ events were thus recorded, each event being constrained by the requirement that the kinetic energy of the light fragment had to be greater than $106 \mathrm{MeV}$. Some of the preliminary data have previously been presented at the Santa Fé Conference [9]. The data presented below will be discussed essentially in terms of mass, charge and energy resolution.

The mass-ratio data correspond to fragments with an emission angle $\theta<24^{\circ}$. Figure 2 shows,

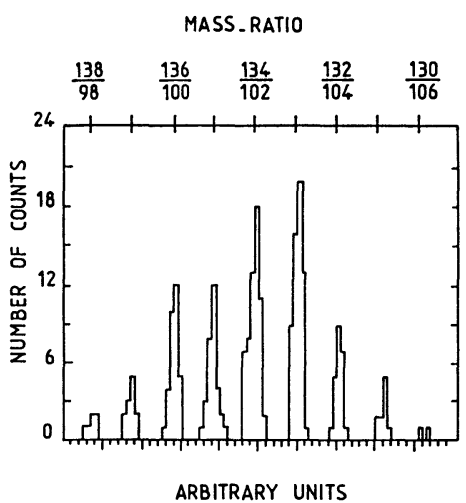

Fig. 2. - A portion of the mass-ratio distribution in a $180 \mathrm{keV}$ window placed at a light fragment kinetic energy of $112 \mathrm{MeV}$. 
as an example, a portion of the mass-ratio distribution for a $180 \mathrm{keV}$ window placed at a light fragment kinetic energy of $112 \mathrm{MeV}$. As can be seen, the mass-ratio separation is total. Each peak has a FWHM of about $0.3 \mathrm{amu}$ which corresponds to a kinetic energy resolution of $450 \mathrm{keV}$ (convolution of the energy resolutions relative to both fragments). At this stage, it is not possible to separate the respective contributions of the target and the chamber. But, as mentioned above, the effective kinetic energy parameter is inferred only from the kinetic energy of the fragment emerging from the deposit side, thus an appreciable improvement of the energy resolution can therefore be expected if one were to decrease the deposit thickness while simultaneously increasing the neutron flux. The limit of the chamber resolution could be reached. According to Oed et al. [6], this limit could be better than $100 \mathrm{keV}$.

The excellent mass-ratio resolution allows one to determine with a good degree of confidence the threshold for the first neutron emission. Clearly, the kinetic energy shift introduced by the sudden loss of one mass unit in one of the two fragments creates a shift on the mass-ratio corresponding to about one-half unit. As an example, figure 3 shows a portion of the mass-ratio distribution for a $180 \mathrm{keV}$ window placed at a light fragment kinetic energy of $108.5 \mathrm{MeV}$. Two secondary peaks are clearly visible between the primary peaks. Such separation is of course rapidly damped by the fragment recoil effect associated with an increase in the mean energy of the emitted neutron as the fragment's excitation energy increases.

For each carefully identified mass-ratio, investigation of the charge-ratio distribution as a function of TKE has been undertaken. As a first result, figure 4 shows the $R_{1} / R_{2}$ distributions obtained for five $320 \mathrm{keV}$ windows placed at differents values of TKE for $A_{1} / A_{2}=104 / 132$. Despite poor statistics due to the strong angular selection as well as the narrowness of the energy window, it can be seen that the charge-ratio resolving power is quite good. The angular selection of the fragments plays of course a fundamental role : the stronger the selection, the better the resolving power. We feel that, for fragments selected around $\theta=0^{\circ}$ a quasi-total separation could be achieved, at least for the highest kinetic energies. Another observation can be made from figure 4 : the position as well as the spacing between two adjacent peaks change slightly with TKE : consequently the resolving power tends to decrease with TKE but the correlated fragment yield increase permits a better angular selection and thus maintains a good resolving power.

The contribution of the different charge-ratios as a function of TKE is very clearly seen in figure 4. For the highest values, there is a unique charge-ratio, namely the one which makes maximum the $Q$-value. According to the Wapstra-Audi tables [10], this ratio is identified as 50/42.

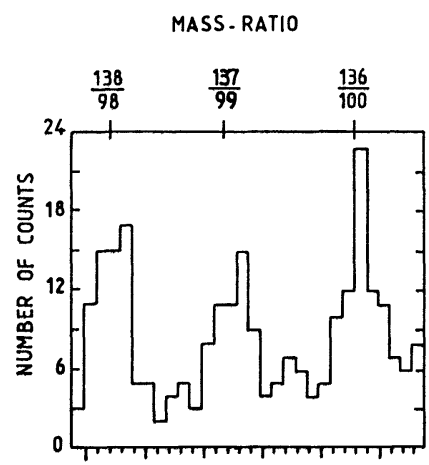

ARBITRARY UNITS

Fig. 3. - A portion of the mass-ratio distribution in a $180 \mathrm{keV}$ window placed at a light fragment kinetic energy of $108.5 \mathrm{MeV}$. 


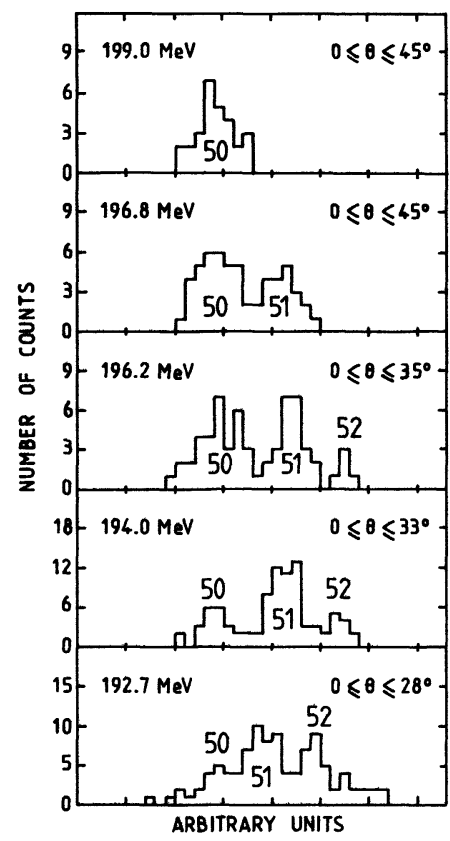

Fig. 4. - Charge-ratio distributions obtained for the mass-ratio 104/132 and for different values of TKE (angular selection and heavy fragment charge assignation are also indicated).

When TKE is decreased, another charge-ratio comes into play, corresponding effectively to the second highest $Q$-value. It is quite important to note that this latter fragmentation is formed by two $N$ odd $Z$ odd fragments ${ }^{104} \mathrm{Nb}_{41}{ }^{1{ }^{132}} \mathrm{Sb}_{51}$. In other words, one neutron pair and one proton pair of the fissioning nucleus had to be broken despite a very low total excitation energy of the scission configuration. More generally the sequence of appearances of the different chargeratios seems to reflect quite well the sequence of the $Q$-values. In other words all the energetically allowed fragmentations are effectively produced.

It would be premature to draw definite conclusions before analysing the whole set of data on an appreciably increased number of events.

\section{References}

[1] Diven, B. C., Martin, H. C., Tasckek, R. F. and Terrell, J., Phys. Rev. 101 (1956) 3.

[2] Guet, C., Asghar, M., Perrin, P. and Signarbieux, C., Nucl. Instrum. Methods 150 (1978) 189.

[3] Signarbieux, C., Montoya, M., Ribrag, M., Mazur, C., Guet, C., Perrin, P. and Maurel, M., J. Physique Lett. 42 (1981) L-437.

[4] Clerc, H. G., Lang, W., Wohlfarth, H., Schmitt, K. H. and Schrader, H., in Proc. 3rd. Int. Conf. on Nuclei far from stability, Cargèse 1975 (CERN 75-13, Genf) 1976, p. 509.

[5] Mantzouramis, G. and Nix, J. R., Phys. Rev. C 25, no 2 (1982) 918.

[6] Oed, P., Geltenbort, P., Gönnenvein, F., Manning, T. and Souque, D., Nucl. Instrum. Methods 205 (1983) 455.

[7] Oed, P., Geltenbort, P. and Gönnenvein, F., Nucl. Instrum. Methods 205 (1983) 451.

[8] Geltenbort, P., PHD thesis (Tubingen University-F.R.G.) 1983.

[9] Trochon, J., Simon, G., Behrens, J. M., Brisard, F. and Signarbieux, C., Proc. Int. Conf. on Nuclear data for basic and applied Science Santa-Fé, May 1985.

[10] Wapstra, A. H. and Audi, G., Nucl. Phys. A 432 (1985) 1. 